1. The demand for oxygen-carrying elements of the blood increases directly with the altitude.

2. In normal individuals this requirement is met through an increase in the red blood corpuscles and hemoglobin in from three to five weeks-the normal acclimatization.

3 . This power of adaptation is diminished or wanting in certain individuals.

4. Deficient acclimatization results in oxygen want or relative anemia.

5. As a result of diminished or limited oxygen supply, the increased excitability or irritability of the nerve structures may be explained.

6 . If by therapeutic or other means, the blood forming mechanism can be stimulated into activity, individuals should find no more difficulty in living tranquil lives in the high altitudes than at the sea levels.

325 Mack Building.

\section{COMPLICATIONS AND SEQUELAE OF THE OPERATION FOR IN- GUINAL HERNIA}

AN ANALYSIS OF ONE THOUSAND AND FIVE HUNDRED CASES AT THE MASSACHUSETTS GENERAL HOSPITAL*

\section{LINCOLN DAVIS, M.D.} BOSTON

The operation for the repair of inguinal hernia is rightly regarded as one of the most satisfactory and simple of surgical procedures. The operative technic has become practically standardized for the routine case, the general principles of closure of the canal being accepted with difference of opinion only as to the merits of transplantation, or nontransplantation, of the spermatic cord. Special and unusual cases, particularly large, direct hernias, may require special measures, such as transplantation of the rectus muscle, or sheath, or implantation of fascia lata.

Large series of cases have been reported by individual operators of great experience, showing a very high percentage of cures and a practically negligible death rate. Coley ${ }^{1}$ reports 3,100 cases of inguinal hernia in which operation was performed at the Hospital for Ruptured and Crippled from 1891 to 1912, with twenty-eight recurrences, less than 1 per cent. of the total number. Out of 3,383 cases of hernia of ail kinds in which operation was performed there were six deaths, a mortality of 0.17 per cent.

The statistics of Wolfler's clinic at Prague comprise 1,460 nonincarcerated inguinal hernias in which operation was performed between 1895 and 1910, with eight deaths, a mortality of 0.63 per cent. Relapses after operation were estimated at from 5 to 8 per cent.

The dissemination of such favorable modern statistics as these, together with improvements in general anesthesia, the introduction of spinal anesthesia, and wide adoption of local anesthesia have greatly extended the field of this operation. An increasing number of the ruptured are insistently demanding surgical relief from the uncertainties and annoyances of truss treatment.

Patients who a few years ago would have been rejected as unfit surgical risks on account of age,

* Read before the Section on Surgery, General and Ablominal, at the Sixty-Seventh Annunl Session of the American Medical Association, Detroit, June, 1916.

1. Coley: Keen's Surgery, vi infirmity or intercurrent disease are now readily accepted for this operation, until it is beginning to be a question if the pendulum is not already swinging too far. At any rate, it seems an opportune time to check up results, to audit, as Codman would express it, our hernia accounts.

The 1,500 cases which form the basis of this report were consecutive cases of inguinal hernia in which operation was performed at the Massachusetts General Hospital from October, 1908, to December, 1914. Definitely strangulated hernias are not included, but cases of incarceration without acute symptoms are counted.

In considering these statistics, it must be borne. in mind that the operations were performed by a large number of surgeons of varying degrees of experience, no less than seventy-five individual operators. There were 1,093 operations done by members of the visiting staff, the largest number to the credit of any one operator being 117 , and the smallest, $1 ; 663$ operations were done by fifty-three different members of the junior house staff.

The patients also showed extreme variation as to age, physical condition and social condition, as would be expected in a large general metropolitan hospital. Comparison of such statistics with those of single operators dealing with somewhat selected material cannot but be disadvantageous.

The youngest patient was 10 months of age, the oldest 77 years. There were ninety patients between 1 and 10 years of age. Eight patients were over 70 . The largest number of cases fell in the decade between 20 and 30 , namely, 397

There were 1,388 males and 112 females. In 1,244 cases the hernia affected one side only; in 256 it was double. In eighty-eight cases the hernia was direct, in the others indirect. No cases of strangulated hernia were included. In ten cases the hernia was of enormous size. In sixty-nine cases the hernia was complicated by undescended testicle. There were nine cases in which the bladder was contained in the sac. In one of these cases there was a large stone in the bladder contained in the hernial sac. The appendix was found in the sac eight times, and removed in the course of the operations forty-six times. There were seven cases of sliding hernia. There was hydrocele present in forty cases, marked varicocele in twenty-six cases. In fifty cases there had been a-previous operation for hernia, with recurrence.

In the 1,500 cases there was a total of 1,756 operations, counting double hernia as two operations. In the male cases the Bassini technic was employed 834 times, Ferguson 764, and Halstead fifteen, with twenty-four cases of varying and miscellaneous technic.

In sixteen cases in which the hernia was complicated by ectopic testicle, orchidectomy. was performed. In fifty cases the undescended testicle was brought down into the scrotum; in one case it was dropped back into the peritoneal cavity. In nine cases orchidectomy was clone in the course of the operation on account of tuberculosis, gumma or other lesion of the testicle.

In many cases operations for other conditions, such as gallstones, ventral hernia, stricture of urethra, etc., were done at the same sitting.

The anesthesia was general in 1,319 cases. Spinal anesthesia was used in eighty-nine cases. Spinal anesthesia was supplemented by general in nine cases, and local in six cases. Local anesthesia was used alone in seventy-five cases. There was one case of rectal anes- 
thesia, and one case in which the anesthetic was not stated.

Accidents of operation were reported as follows: Injury to the bladder in two cases, with immediate suture, without ill effect. The vas deferens was reported cut seven times; in one case end-to-end suture was attempted. In two cases a small nick was made in the bowel and immediately sutured without bad result. There were no cases of injury to the iliac vein.

There were eight deaths in this series, a mortality of 0.53 per cent. In two of these fatal cases there were complicating conditions present which were largely responsible for the result. One case was that of a man of 63 with diabetes mellitus, having a scrotal hernia and symptoms of severe cystitis. Under spinal anesthesia a suprapubic cystotomy was done; it was then found that there was a hernia of the bladder through the inguinal canal. As the bladder could not - be withdrawn through the hernial opening, the inguinal canal was opened and a stone was found in the portion of the bladder lying in the hernial sac. The stone was removed, the inguinal canal repaired and bladder closed about a suprapubic drain. The patient died of sepsis. This unusual case might, I think, fairly be left out of hernia statistics.

In the other complicated case a ventral hernia was repaired at the same sitting; general peritonitis developed, with fatal result. Necropsy showed that the peritonitis developed from sepsis in the ventral hernia wound. In this case it is manifestly unfair to burden the inguinal hernia operation with the fatal result. This leaves six cases of straight hernia with fatal issue. In three cases death occurred suddenly, and unexpectedly, on the fifth, thirteenth and fourteenth days, respectively, and was ascribed clinically to pulmonary embolism. There were no necropsies.

In one case the patient, a feeble man of 69, with double hernia, gradually petered out and died comatose on the tenth day.

In another case of double hernia a large hematoma developed which was subsequently drained. A secondary operation was performed on the eighth day on account of abdominal pain, distention and vomiting, with high temperature. Perforated ulcers of the colon were found, with general peritonitis. Necropsy showed no direct connection between the hernia operation and the peritonitis, which was evidently the result of perforation of ulcers of the colon.

In the last case death resulted on the eighth day, with symptoms of vomiting, distention and jaundice. Necropsy showed focal pneumonia, and extensive hemorrhage into the mesentery and retroperitoneal tissues.

Nonfatal postoperative complications of more or less severity developed in 438 cases, or 28 per cent.; some of these were trivial. In many cases the complications were multiple.

\section{COMPLICATIONS}

Sepsis.-In 158 cases the wounds failed to heal by first intention. In ninety-three cases the sepsis was trivial, consisting either of a stitch abscess, or more often a collection of serum which required draining. In many of the latter cases probably no infection was present; cultures were not made. In sixty-five cases there was frank pus in the wound, under 4 per cent. of the total number of wounds.

Of the seventy-five cases in which operation was performed with local anesthesia, sepsis developed in the wounds in thirteen cases, or 17 per cent.
Of the eighty-nine cases in which operation was performed with spinal anesthesia, there were nine septic wounds, or 10 per cent.

Hematoma.-In 112 cases the development of a hematoma was mentioned in the record; in twentyseven of these cases it was stated to be slight. Marked and extensive ecchymosis was noted in two cases; in one of these it extended to the chest wall. In both cases it subsided spontaneously.

Subsequent orchidectomy became necessary in five cases, once on account of tubercilosis, and once on account of sloughing of a transplanted undescended testicle.

Complications in the Respiratory Tract.-These were of common occurrence, namely, 138 cases $(9.2$ per cent.) distributed as follows: Frank pneumonia was reported in seven cases, questionable pneumonia in one. Bronchitis was recorded in forty-eight cases; postoperative cough, with slight temperature, in fortythree; pulmonary infarct, two; pleurisy, one; colds, six, and tonsillitis, thirty.

Respiratory complications were by no means abolished by the use of spinal or local anesthesia; this probably was partly due to the fact that these forms of anesthesia were selected on account of chronic bronchitis or cough on the part of the patient. At any rate, respiratory complications occurred in twenty-five of the 170 in which spinal or local, or a combination of the two were used, nearly 15 per cent. Of these, one case of pneumonia and one fatal case of pulmonary embolism followed spinal, and one nonfatal pulmonary infarct local, anesthesia.

Miscellaneous.-There were many miscellaneous complications distributed as follows: otitis media, two; measles, one; cholangeitis, one; persistent hiccup, three; phlebitis, three; acidosis, two; pyelitis, one; ataxic paraplegia, one (this was not a case of spinal anesthesia). There were three cases with marked postoperative mental symptoms; one with delirium, and two with hallucinations and attempted suicide. The latter were both cases of spinal anesthesia.

\section{END-RESULTS AND RECURRENCE}

End-results were obtained through the routine follow up system of the hospital, no special effort being made to trace cases for this report. The hospital system consists of sending a letter to each patient, one year from date of discharge, requesting return to the hospital for examination; if this is impossible, report of condition by letter is requested.

As already stated, eight patients died in the hospital after operation. Data subsequent to discharge were obtained in 754 cases, just over 50 per cent., in which 140 patients reported by letter and 614 reported in person for physical examination. Eleven patients died of intercurrent disease during the year; 577 were reported unequivocally well, or cured, 38 per cent. of the total number operated on, and 76 per cent. of those whose subsequent histories are known. Ninety-nine patients are classed as relieved; fifty of these were cured of the hernia but complained of subjective symptoms more or less closely associated with the wound. The commonest complaint was of pain in the wound, especially when working or lifting. Two complained of persistent numbness in the inguinal region. Of the others in the relieved class, seventeen patients had no actual recurrence of hernia but a bulge in the region of the scar; eight had marked varicocele; five were cured of the hernia for which they were operated on, 
but subsequently developed hernia elsewhere; three had atrophy of the testicle; two had keloid in scar; one had a persistent sinus; one developed adenocarcinoma of the sigmoid, and one was cured of hernia but had incontinence of urine. There were six cases of miscellaneous and unclassifiable complaints.

Fifty-nine patients had definite recurrence within the year; five of these had double recurrences; two are known to have recurred after the lapse of a year, making a total of sixty-six recurrences, or 3.7 per cent. of the total number of operations performed, and $8+$ per cent. of the number of cases traced. There were six cases in which there was questionable recurrence. In two of these, relapse was claimed by the patient but could not be found by the examiner. If these six cases are counted as recurrences, the percentage is raised to 9 per cent. of the cases traced.

Of these recurrences, twenty-six, or 3.1 per cent., followed the Bassini operation, and thirty-four, or 4.4 per cent., the Ferguson.

In the sixty-seven cases of recurrence or questionable recurrence, postoperative sequelae were distributed as follows: cough, eleven cases; hematoma, nine, and sepsis, four.

In the 112 female cases there were only two recurrences.

Of the eighty-eight direct hernias there were recurrences noted in seven, or 7.9 per cent., or 15 per cent. of the direct hernia cases traced.

There were only two relapses out of seventy-five cases in which operation was performed with local anesthesia, 2.5 per-cent. of recurrences; but it must be borne in mind that these were for the most part the less severe types of cases.

Simmons found in his study of hernia that recurrence practically always takes place within six months after operation. This is undoubtedly true for the great majority of cases, but like all generalizations is subject to exceptions. In two of our cases counted among the recurrences, the patients were reported well at the end of the first year. Others which we do not know about may also have relapsed since the expiration of the year.

\section{SUMMARY AND CONCLUSIONS}

In summing up this statistical study of inguinal hernia, I desire to emphasize the following points:

The results of operation are on the whole good, better than might be expected under the conditions. The operation, however, has a definite, though low, mortality rate, and should not be undertaken in the old and the infirm without good reason.

Postoperative cough, hematoma and sepsis are important factors in the incidence of recurrence, but the latter complication seems to play a lesser rôle than is generally assigned to it.

A strikingly lärge number of patients anatomically cured complain of pain, probably due to nerve traumatism.

General anesthesia is still best in the routine case. Local anesthesia is very satisfactory, and has a wide application in cases in which inhalation anesthesia is contraindicated, but carries a slightly greater risk of sepsis, and hence probably of recurrence too, althongh the latter conclusion is not borne out by our figures. Spinal anesthesia, on account of its greater danger and serious sequelae, should have little place in this operation.
Careful study of the results in this series of cases reaffirms the importance of the well recognized surgical principles of clean anatomic dissection, conservation of nerve supply, high closure of sac, securely accurate coaptation of tissues without constriction, and complete hemostasis, in the attainment of success in the operation for inguinal hernia.

\section{ABSTRACT OF DISCUSSION}

Dr. Henry O. Marcy, Boston: Tracing the development of the male during the late fetal period we note the extrusion of the testicle from the abdomen and the carrying with it of the peritoneal covering investing the vessels and vas deferens, making up the spermatic cord. Normally, the peritoneum is so closed in about the structures at the exit from the abdomen that only by pulling on the cord do we note the slight depression on the peritoneal surface, which lines the abdomen at this point. When the opening through the abdominal wall is imperfectly closed there results a more or less marked depression of the peritoneum described by Cloquet as normal anatomy and called by him the "infundibular process of the peritoneum." This defective closure of the internal ring is the usual cause of indirect hernia, although it may not occur until an advanced age. When normally developed the intraabdominal pressure is at right angles to the canal and firmly closes it. The reconstruction of the canal to its normal size and obliquity is the sine qua non in the cure of hernia. In 1870 I operated on a large strangulated hernia and closed the wound with buried catgut sutures. The cure remained permanent. My first publication for the cure of hernia was in November, 1870 . I instituted a long series of studies on animals to determine the rôle of buried. animal sutures and published many articles on their advantages. I early emphasized the reconstruction of the ingtinal canal, to its normal size and obliquely as safe, easy and permanent. The cure of hernia was established on a firm basis. In 1882 I first obtained tendons from the tail of the kangaroo and introduced their use as a suture material in every way superior to catgut. Corroboration of the principles and practice which $I$ have so long advocated is now ample. All aseptic wounds closed with buried aseptic tendon sutures will remain aseptic and in well vitalized structures will be followed by primary union. The aseptic absorbable animal suture, preferably tendon, I consider my best contribution to surgery and the cure of hernia is dependent on their wise use. For a long period it has been a far too common custom to accredit to European writers much that belongs to our profession. To Bassini, honor is due for his excellent work, but in method, experience and publication he is antedated by a number of American surgeons. At last the cure of hernia should be called the "American method." With all the emphasis of an earnest conviction, I commend to every aseptic surgeon familiarization with the methods of wound closure by means of buried absorbable sutures, and not alone predict their early general adoption but that, in importance, they have the first place in the technic of modern aseptic wound treatment.

Dr. Daniel Nathan EIsendrath, Chicago: Hernias in hospitals are generally operated on by the house men and careful supervision is not given to a series of operations by one operator. I was very much struck with one point made: that was the frequency of sepsis in cases with local anesthesia. I have had that experience in my early cases, but since $I$ have diluted the epinephrin $I$ have not had experiences of that kind. I think we use epinephrin as a local anesthesia too strong and we have a reduction in the vitality of the tissues which favors intervention. Instead of using 4 or 5 drops of a $1: 1,000$ solution to the ounce I seldom use more than 1 drop. A second point to which I wish to call attention is some of the causes of recurrences. I think we are very apt to forget that there are in a large number of hernias a series of diverticular sacs. I mean the sacs which come out from the interral ring and form a double sac, i. e.. a short trouser leg and a long trouser leg. Many of 11 : were formerly satisfied with dissecting up and ligating the neck of the sac. We think we are ligating high up. When- 
ever I find one having on the outer aspect a great deal of fat $I$ put my finger in and see if there is a diverticulum, $i$. e., a pantaloon sac. Another cause is the comparative frequency of the concomitant occurrence of the direct and indirect hernia on the same side, parallel with each other and we are apt to overlook either the direct and the indirect. The next point is one Dr. Davis calls attention to, viz., the frequency of pain following hernia operations, and I have learned to be careful not to include any nerve fibers in my sutures. At the Michael Reese Hospital in Chicago, we uniformly do the Andrews operation of imbrication. It is the ideal operation in the type of cases of which we see so many, those with the very weak conjoined tendons. Our best result is in transplanting the cord invariably. I have not made an exception cxcept in cases of undescended testes. The reason why this Andrews operation with the transplant of the cord is the ideal operation is that if you do not transplant the cord you have at the lower end a space that represents the pubic bone. You must make allowance for hard bony material with suture material. If you do transplant the cord you can work the many muscle tissues. I think that is the reason why a great many of these cases have recurrences, for the 34 per cent. of the total number of recurrences following the Ferguson operation.

Dr. Edward W. Lee, New York: To what extent, if any, does Dr. Davis consider that traumatic hernias exist? Is there such a thing as traumatic hernia? Is there not a condition that predisposes to hernia that would let hernia be produced? I ask this question because in connection with a former paper the workman's compensation law and medicolegal cases this question is becoming one that somebody has got to decide very definitely to determine whether we actually have what may be called traumatic hernia.

Dr. Lincoln Davis, Boston: In regard to the question just asked: I do not know. I have no data on traumatic hernia. It is my belief that there is a predisposing factor present in all these cases, probably a pouch in the peritoneum which enables the bowel to come through suddenly, but I really have not gone into that question. I agree entirely with what Dr. Eisendrath said in regard to the double sac. I think that is a very important matter, and I have frequently found a direct hernia accompanying indirect and $I$ think it is the direct hernia which is the problem to cure. Particularly in those direct hernias, I think Dr. Andrews' operation of imbrication and overlapping the layers is of great value. As to sepsis in local anesthesia, I think our figures are altogether too high and we have perhaps been a little slow in taking up local anesthesia. I think we are going to use it more and more. I am a little bit in doubt myself as to whether you can do quite as secure a closure under local as under general anesthesia. I think the infiltration of the tissues around the neck of the sac interferes with the most accurate and firm closure of the sac. We have not followed enough cases that have been done under local anesthesia to be convinced on that point. Dr. Marcy showed how the obliquity of the canal is one facto in preventing hernia and that is the condition that you wish to restore and it is for that reason that the direct hernia is so much more of a problem than the indirect. I found in these statistics which I have given you, that there was very little difference in the percentage of recurrence between the so-called Bassini and Ferguson operations. It was a little less than 1 per cent., I believe.

Loss of Weight of Musk.-Most of us remember that in our high school days the odor of musk furnished an illustration of matter in a finely divided molecular condition. We were taught that musk may give off odor indefinitely, meanwhile retaining its fragrance and weight unimpaired, or, at least, that any hypothetical loss of weight due to the subtraction of odoriferous particles was not susceptible of demonstration by tize most delicate scales. According to the Journal of the Franklin Institute, however, this has been experimentally disproved. It is stated that musk, exposed for about seven months to a current of dry air, loses weight. When the loss of weight has ended, the musk is odorless, and its fragrance cannot be restored by exposure to damp air.

\section{THE PREVENTION OF OBSTRUCTION OF THE PASSAGE OF GAS}

FOLLOWING OPERATIONS ON THE COLON*

\section{A. J. OCHSNER, M.D. CHICAGO}

A review of our work in surgical operations on the colon, as well as a review of the literature on this subject, impresses one most forcibly with the fact that, barring sepsis, which is now almost never encountered, and necrosis due to interference with the blood vessels or tension, both of which conditions can readily be avoided, the greatest danger to the patient comes from tension caused by obstruction to the passage of gas following colon operations. It seems, consequently, wise in performing any operation on the colon to plan the operation in such a manner as to make it impossible for a sufficient amount of gas to accumulate above the seat of the operation to endanger the success of the operation. If the surgeon has this fact definitely in view, it is usually not difficult to plan some provision which will be effective in preventing this source of danger. Several of these provisions have been fully described by a number of surgeons, but so far as I have been able to determine, the subject as applied to the entire colon has not received a due amount of consideration in surgical literature, and it

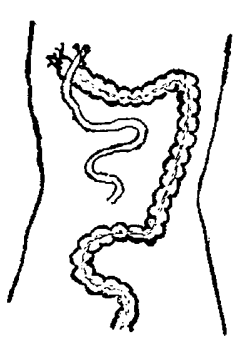

Fig. 1.-F r a $\mathrm{n} \in \mathrm{i}$ $R$ ed e $r$ operation: anastomosis between ileum and colon.

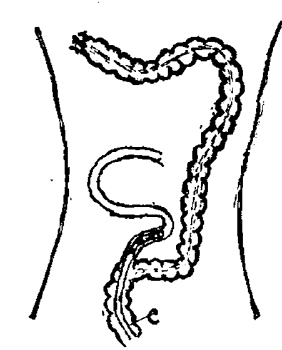

Fig. 2.-Excision of cecum: anastomosis between ileum and drain extending into drain seems worth while to undertake the task of presenting this subject at the present time together with diagramm a $\mathrm{t}$ i $\mathrm{c}$ drawings of the actual steps which we have employed in our clinic for this purpose.

Francis Reder has described an ingenious method for preventing pressure from gas in cases in which the cecum has been removed and in which the ileum is implanted into the transverse colon, as shown in Figure 1 . The end of the ileum is passed out through a buttonhole in the abdominal wall about $10 \mathrm{~cm}$. beyond the point at which the anastomosis between the closed end of the transverse colon and the end of the ileun is made. A tube is inserted into the free end of the ileum which will permit the gas to escape which may accumulate in the ileum, until perfect healing has taken place between the ileum and the transverse colon, and until the enterostomy opening functionates perfectly. When the drainage tube is removed, the remaining wound will heal spontaneously. If there is any difficulty about the healing of this wound, this can be stimulated by touching the lumen of the projecting intestine with the actual cattery.

In case the distance between the free ends of the ileum and the transverse colon is so great that an anastomosis cannot be accomplished without the use of a degree of tension which might endanger the vitality of the intestine, the same operation may be performed with equal safety by closing the free end

* Read before the Section on Obstetrics, Gynecology and Abdominal Surgery at the Sixty-Seventh Annual Session of the American Medical 